\title{
ON ALGORITHMS FOR BROWNIAN DYNAMICS COMPUTER SIMULATIONS
}

\author{
ARKADIUSZ C. BRAŃKA \\ Institute of Molecular Physics, Polish Academy of Sciences, \\ Smoluchowskiego 17/19, 60-179 Poznań, Poland
}

\begin{abstract}
Several Brownian Dynamics numerical schemes for treating stochastic differential equations at the position Langevin level are analyzed from the point of view of their algorithmic efficiency. The algorithms are tested using model colloidal fluid of particles interacting via the Yukawa potential. Limitations in the conventional Brownian Dynamics algorithm are shown and it is demonstrated that much better accuracy for dynamical and static quantities can be achieved with an algorithm based on the stochastic expansion and second-order stochastic Runge-Kutta algorithms. Mutual merits of the second-order algorithms are discussed.
\end{abstract}

\section{INTRODUCTION}

Dispersed systems such as polymer solution and colloidal liquids are often governed by a set of many stochastic equations in which the influence of the large number of solvent molecules on polymer or colloidal particle is represented by random forces and frictional terms. The complexity of such systems prohibits exact analytic treatments in all but the most idealised of cases (e.g., infinitely dilute systems). As the result, various problems in dispersed systems can be solved almost exclusively by computer simulations. Comparing to the well established techniques for solving deterministic equations of motion the methods for solving stochastic equationas, offten called stochastic dynamics (SD), are considerably less developed.

Even in the most simple form of SD called the Brownian dynamics (BD) the main tool for solving the equations is the low-order algorithm by Ermak and McCommon [1] [2], This technique is at the level of the first-order Euler method for ordinary differential equations and requires veiy small time step to produce stable and sufficiently accurate results. Thus, the method is simple, straightforward but quit inefficient (usually up to two orders of magnitude longer runs are needed in the BD than in the molecular dynamics (MD)). The way to make the BD method more efficient is to apply an appropriate second or higher-order algorithms. Unfortunately, only a few proposals has been made, with little concern about its ability for treating efficiently large physical many-body systems, and as shown below none of them can be considered as fully satisfactory.

Most of them are Runge-Kutta like algorithms with some stochastic terms. Algorithms along this line has been proposed e.g., by Helfand [3], Iniesta and Torre [4], and recently one rigorously developed for the one-variable case by Honeycutt [5], All these methods, like the deterministic Runge-Kutta methods, require more than one evaluation of the particle force per time step, which clearly reduces its efficiency. From the other hand they employ larger time step and it has been argued that stochastic Runge-Kutta (SRK) approach gives, more accurate results than the conventional $\mathrm{BD}$ (for the same amount of computer time) [4]. 
The algorithm which needs only one evaluation of the force per time step was proposed by van Gunsteren and Berendsen [6], In the limit of the large friction (i.e., most important from point of view of BD applications) the algorithm has particulary simple form, and has been used to model, for example, polymer dynamics in solution [7].

In the present work we shall consider, from the point of view of the BD algorithms, the iterative solution of stochastic differential equations and make the efficiency comparison between different BD algorithms. The basic BD algorithms are considered in section 2 and in section 3 a numerical test is discussed. Conclusions are in section 4.

\section{THE ALGORITHMS}

The motion of $\mathrm{N}$ interacting particles of solute (e.g., colloidal particles), for many purposes, is adequately described by the position Langevin equation,

$$
\frac{d r_{i \alpha}}{d t}=\frac{D}{k_{B} T} F_{i \alpha}+D^{1 / 2} \xi_{i \alpha}
$$

where $i=1, \ldots, \mathrm{N}$ labels the particles and $\alpha, \beta, \gamma \mathrm{fl}$ indicate the cartesian coordinates $(x, y, z$ or $1,2,3) . \xi(t)$ is the gaussion white noise process, $D$ is the free-particle diffusion constant, $k_{B}$ is Boltzmann's constant, and $T$ is the temperature. $F_{i \alpha}$, is the net force acting in the direction $\alpha$ on the particle, i, derived from the interparticle potential $U\left(r^{N}\right)$ usually represented as a sum of pairwise additive direct interactions $V(r)$ between the particles,

$$
\mathbf{F}_{i}=-\nabla_{i} U=-\nabla_{i} \sum_{j \neq i}^{N} V\left(\left|\mathbf{r}_{i}-\mathbf{r}_{j}\right|\right)
$$

The set of equations (1) constitutes stochastic description which is equivalent to the Smoluchowski equation without hydrodynamic interactions. The stationary solution of the Smoluchowski equation is the canonical ensemble distribution,

$$
P\left(\mathbf{r}^{N}, \infty\right)=\frac{1}{Z} e^{-U / k_{B} T},
$$

where $\mathrm{Z}$ is the partision function and thus the time averages produced by the equations (1) are the canonical ensemble averages. From the Smoluchowski or the Langevin equations the short-time behaviour of time correlation functions can be estimated [8], Explicit results for the form of the Cartesian components of the mean square displacement are aviable to fourth order in time,

$$
\left\langle\Delta r_{i \alpha}^{2}\right\rangle=2 D t-\frac{D^{2}}{T}\left(\frac{\partial^{2} U}{\partial r_{i \alpha}^{2}}\right) t^{2}+\frac{D^{3}}{3 T^{2}} \sum_{j=1}^{N} \sum_{\beta=1}^{d}\left(\left(\frac{\partial^{2} U}{\partial r_{i \alpha} \partial r_{j \beta}}\right)^{2}\right) t^{3}-B t^{4}+O\left(t^{5}\right),
$$

where $d$ denotes the dimensionality of the system, There and in the following means $k_{B} T$, and $B$ containes higher derivatives of the total potential energy and the explicit form of this term is irrelevant here.

The conventional Brownian Dynamics algorithm (CBD) solves the equations (1) according the following update scheme, 


$$
r_{i \alpha}(\Delta t)=r_{i \alpha}+\frac{D}{T} F_{i \alpha} \Delta t+W_{i \alpha}
$$

where $\Delta t$ is the time step, $r_{i \alpha}=r_{\mathrm{i \alpha}}(0)$, and $W_{i}$ is a normally distributed random force with zero mean and $\left(W_{\mathrm{i} \alpha} W_{\mathrm{j} \beta}=2 D \Delta \mathrm{t} \delta_{\mathrm{ij}} \delta_{\alpha \beta}\right.$. Van Gunsteren and Berendsen proposed the following algorithm (GB),

$$
r_{i \alpha}(\Delta t)=r_{i \alpha}+\frac{D}{2 T}\left(2 F_{i \alpha}+\Delta t \dot{F}_{i \alpha}\right) \Delta t+W_{i \alpha},
$$

where the time derivative of the force is conventionally approximated by

$$
\dot{\mathbf{F}}=[\mathbf{F}(t)-\mathbf{F}(t-\Delta t)] / \Delta t
$$

The second order stochastic Runge-Kutta algorithm (SRK) updates particle positions

$$
r_{i \alpha}(\Delta t)=r_{i \alpha}+\frac{D}{2 T}\left(F_{i \alpha}^{a}+F_{i \alpha}^{b}\right) \Delta t+W_{i \alpha},
$$

calculating the forces in two stages $\mathbf{F}_{i}^{a}=\mathbf{F}_{i}\left(\boldsymbol{r}^{N}\right)$, and then $\mathrm{F}_{i}^{b}=\mathrm{F}_{i}\left(\mathrm{R}^{N}\right)$, at $\mathbf{R}_{i}=\mathbf{r}_{i}{ }^{-}+D \mathbf{F}_{i} \Delta t+\mathbf{W}_{i}$. For a general stochastic differential equation such as Eq. (1) the following expansion for the Cartesian components of the particle position holds [9],

$$
\begin{aligned}
r_{k \alpha}(\Delta t) & =r_{k \alpha}+W_{k \alpha}+\frac{D}{T} F_{k \alpha} \Delta t+\sum_{j=1}^{N} \sum_{\beta=1}^{d} \frac{\partial F_{k \alpha}}{\partial r_{j \beta}} K_{j \beta}+\frac{1}{2} \Delta t^{2} \sum_{j=1}^{N} \sum_{\beta=1}^{d} \frac{\partial F_{k \alpha}}{\partial r_{j \beta}} F_{j \beta}+ \\
& +\frac{1}{2} \sum_{p, j=1}^{N} \sum_{\beta, \gamma=1}^{d} \frac{\partial^{2} F_{k \alpha}}{\partial r_{p \gamma} \partial r_{j \beta}} G_{p j}^{\beta \gamma},
\end{aligned}
$$

where $K$ and $G$ are random numbers involving $W$,

$$
K_{i \alpha}=\int_{0}^{\Delta t} W_{i \alpha}(s) d s, \quad G_{i j}^{\alpha \beta}=\int_{0}^{\Delta t} W_{i \alpha}(s) W_{j \beta}(s) d s
$$

The expansion of Eq. (8) results from integration of Eq. (1), the Taylor expansion for $F$ and its repeated insertion into itself [9], It is important to recognize that the random number $K_{i \alpha}$ is also, like $W_{\text {i } \alpha}$, a Gaussian random number with the following properties,

$$
\left\langle K_{i \alpha}\right\rangle=0,\left\langle K_{i \alpha} K_{j \beta}\right\rangle=\frac{2}{3} D \Delta t^{3} \delta_{i j} \delta_{\alpha \beta},\left\langle W_{i \alpha} K_{j \beta}\right\rangle=D \Delta t^{2} \delta_{i j} \delta_{\alpha, \beta} .
$$

Thus, the term in Eq. (8) involving $K$ is of order $\Delta t^{3 / 2}$. The last term is of order $\Delta t^{2}$ but its nonlinearity does not allow to obtain a more explicit representation and the exact formula is replaced by simpler (local) expressions with the same first moment, $G_{i j}^{\alpha \beta} \approx 1 / 2 \Delta t W_{i \alpha} W_{j \beta}$. In the following the algorithm based on the expansion of Eq. (8) will be called the Stochastic Expansion algorithm, (SE). We shall also consider the SEb algorithm of the order $\Delta t^{3 / 2}$, based on the first four terms in the expansion (8) (the first three terms are just the CBD update scheme). Some formal difference between the SRK and the SE approach can be deduced expanding the $\mathbf{F}^{b}$ force in the SRK scheme [9], The resulting formula is very similar to that in eq.(8) apart of the $\Delta t^{3 / 2}$ terms in which $K_{\mathrm{i} \alpha}$ are approximated by $1 / 2 \Delta t W_{\mathrm{i} \alpha}$. It can be shown that, as far as expansion parameter is small the following SRK-like scheme gives exactly the SE-expansion (8), 


$$
r_{i \alpha}(\Delta t)=r_{i \alpha}+\frac{D}{2 T}\left(F_{i \alpha}^{a}+F_{i \alpha}^{b}\right) \Delta t+W_{i \alpha},+\sum_{j=1}^{N} \sum_{\beta=1}^{d} \frac{\partial F_{i \alpha}^{a}}{\partial r_{j \beta}} S_{j \beta},
$$

where

$$
\left\langle S_{i \alpha}\right\rangle=0,\left\langle S_{i \alpha} S_{j \beta}\right\rangle=\frac{2}{3} D \Delta t^{3} \delta_{i j} \delta_{\alpha \beta},\left\langle W_{i \alpha} S_{j \beta}\right\rangle=0
$$

It is to be noted that $S_{i \omega}$, like $K_{i \omega}$, is a gaussion random number but, unlike $K_{i \alpha}$, is not correlated with $W_{i a}$. The algorithm based on the formula (11) we shall call the SRKb algorithm.

An important quantity enabling us to distinguish various algorithms is the mean-square displacement in a time step, MSD1. Its exact form follows from Eq. (4). The CBD algorithm gives only the trivial linear approximation,

$$
\left\langle\Delta r_{i \alpha}^{2}\right\rangle=2 D \Delta t+\frac{D^{2}}{T}\left\langle\frac{\partial^{2} U}{\partial r_{i \alpha}^{2}}\right\rangle \Delta t^{2}+\delta_{C B D} \Delta t^{2}
$$

and the error in the second order term is

$$
\delta_{C B D}=\frac{D^{2}}{T}\left[\frac{1}{T}\left(\left(\frac{\partial U}{\partial r_{i \alpha}}\right)^{2}\right)+\left\langle\frac{\partial^{2} U}{\partial r_{i \alpha}^{2}}\right)\right] .
$$

For many physical realizations the following relation holds,

$$
\left\langle F_{i \alpha}^{2}\right\rangle=T\left(\frac{\partial^{2} U}{\partial r_{i \alpha}^{2}}\right)
$$

what implies $\delta_{\text {С в }}>0$. Thus, the CBD algorithm always overestimates the MSD1 by

$$
2 D^{2} \Delta t^{2}\left\langle F_{i \alpha}^{2}\right\rangle / T^{2}
$$

The GB algorithm also yields an error in the second order term,

$$
\left\langle\Delta r_{i \alpha}^{2}\right\rangle=2 D \Delta t+\frac{D^{2}}{T}\left\langle\frac{\partial^{2} U}{\partial r_{i \alpha}^{2}}\right\rangle \Delta t^{2}+\delta_{G B} \Delta t^{2}
$$

where

$$
\delta_{G B}=\delta_{C B D}+\frac{3}{2}\left\langle F_{i \alpha}^{2}\right\rangle\left(1-\frac{\left\langle F_{i \alpha}(\Delta t) F_{i \alpha}(0)\right\rangle}{\left\langle F_{i \alpha}^{2}\right\rangle}\right)
$$

As the normalized autocorrelation function is less than unity, the second contribution in the $\delta_{G B}$ is always positive. This means, rather surprisingly, that in general the GB algorithm yields larger errors than the CBD algorithm. The SRK algorithm gives,

$$
\left\langle\Delta r_{i \alpha}^{2}\right\rangle=2 D \Delta t+\frac{D^{2}}{T}\left\langle\frac{\partial^{2} U}{\partial r_{i \alpha}^{2}}\right\rangle \Delta t^{2}+\delta_{S R K} \Delta t^{2},
$$

with an error contribution, 


$$
\dot{\delta}_{S R K}=\frac{D^{3}}{2 T^{3}}\left(F_{i \alpha}-\left\langle F_{i \alpha} F_{i \alpha}^{b}\right\rangle\right),
$$

which is always positive and for small $\Delta t$ can be approximated by the linear term. Thus, the SRK algorithm is the first algorithm to give the correct second order term for MSD1 and yields an underestimation of MSD1 with leading term of order $\Delta t^{3}$. The same is true also for the SRKb algorithm.

Equation (8) yields the following expression for the MSD1,

$$
\begin{aligned}
\left\langle\Delta r_{i \alpha}^{2}\right\rangle & =2 D \Delta t+\frac{D^{2}}{T}\left(\frac{\partial^{2} U}{\partial r_{i \alpha}^{2}}\right) \Delta t^{2}+ \\
& +\frac{D^{2}}{T}\left[\frac{1}{T}\left\langle\left(\frac{\partial U}{\partial r_{i \alpha}}\right)^{2}\right)-\left(\frac{\partial^{2} U}{\partial r_{i \alpha}^{2}}\right)\right] \Delta t^{2}+\delta_{S E} \Delta t^{3} .
\end{aligned}
$$

On the base of the relation (14) the second term of order $\Delta t^{2}$ is equal to zero (for any $\Delta t$ ) and the expansion of Eq. (8), like the SRK algorithm, gives the correct MSD1 with some deviation only in terms of order $\Delta t^{3}$. It should be noticed that the same is true also for the SEb algorithm with an error $\delta_{S E b} \neq \delta_{\mathrm{SE}}$ in terms of order $\Delta t^{3}$.

\section{NUMERICAL CALCULATIONS}

In order to compare the efficiency of the above algorithms with increasing $\Delta t$ and to establish how the MSD1 errors influence the static and dynamic quantities we have considered the dynamics of $N=121$ Brownian particles in two dimensions (2D) interacting via the Yukawa pairwise potential,

$$
V(r)=\frac{V_{0}}{r} \exp [-\lambda(r-1)]
$$

where $V_{0}$ sets the energy scale and $\mathrm{A}$ is the screening parameter characterizing the steepness and range of the potential. The Yukawa potential, being the electrostatic part of the DLVO potential [10], is expected to give a good description of the interaction of a dilute charge-stabilized spherical colloidal suspensions [11].

The basic simulation cell was a square of the area $A$, and usual periodic boundary conditions were applied. In order to make the system suitable for testing various algorithms the simulations were performed at the dense fluid region $(T=1, \quad=N / A=0.5)$ and the interaction potential was chosen to be strongly repulsive, $\lambda=8$.

All quantities are normalized to dimensionless forms, by choosing $\sigma, \sigma^{2} / D$ and $V_{d} / \sigma$ as the characteristic values for length, time and forces. In the calculations, the averages were calculated from simulations of about $10^{3}$ reduced time periods (what is e. g., $10^{7}$ time steps with

$\Delta t=0.0001$ ). In the SE algorithm based on Eq. (8) the two correlated random numbers $W_{i \alpha}$ and $K_{i \alpha}$ were sampled from a bivariate Gaussian distribution. 
Perhaps the most important yet simplest dynamical quantity is the one we shall consider, and that is the time dependent mean square displacement, msd. In the long-time limit it gives the self-diffusion coefficient of the particle. The general behavior of the $m s d$ calculated with the various algorithms is shown in Fig. 1 for a fairly large time step $\Delta t=0.0005$ (for other time steps the results are qualitatively veiy similar). On the scale of the figure the msd curves obtained from the SRK, the SRKb and the SE calculations coincide with the exact curve (i.e., the curve produced by all algorithms in the limit of very small time step). The CBD curve very slowly approaches the exact curve from above. Also the GB curve deviates considerably from the exact curve at short times but converges relatively quickly at longer times (in about 10-15 $\Delta t$ ) to the correct form. Notice that in accordance with Eq. (17) it starts above the CBD curve. The SEb produces correct short and long time limits but slightly bends downward at intermediate times. Therefore, all algorithms reproduce correctly the long-time behavior of the $m s d$, but differ considerably in their ability to reproduce its intermediate and particularly short-time behavior. This is clearly seen in the enlargement in Fig. 1 which illustrates a significant influence of the MSD1 on the short-time behaviour of the msd. In the insert, slight differences between the SRK, the SRKb and the $\mathrm{SE}$ algorithms emerge which become more significant as $\Delta t$ increases. These differences signal that the third order corrections start to play a role for $\Delta t \geq 0.0005$. The SRK algorithm preserves the initial small MSD1 deviation for a long time. The SE method, in contrast, converges quickly to the exact curve. The SRKb method produce results which lie between these two cureves.

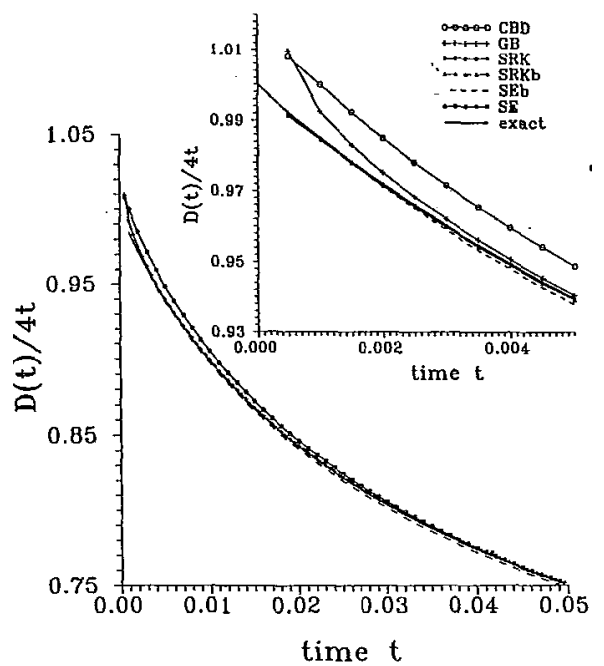

Fig. 1. The mean square displacement, $m s d$, of a 2D Yukawa fluid obtained from the six BD algorithms discussed in the text with time step $\Delta \mathrm{t}=0.0005$. The curve labelled, "exact" is the limit obtained from calculations with $\Delta t=0.00002$. The SE, the SRK, and the SRKb algorithm data coincides with the exact curve. The insert presents an enlargement of the short-time region.

The behaviour of the static quantities obtained by different algorithms at various time steps is illustrated in Fig. 2 where the total energy per particle is shown. The results for the pressure are very similar. It appears from the figure that the best approximation, at given value of $\Delta t$, is achived by the SE method and the worst (as expected) is by the CBD route. For $\Delta t \leq 0.0005$ the results produced by the SRK and the SRKb algorithms are practically as good as the SE (within the error bars all they coincide with the exact values). For the larger time steps the diviations from the exact value becomes more significant. 
Fig. 2. Energy of the Yukawa fluid vs. time step from the different algorithms. The exact value is estimated to be 0.4345

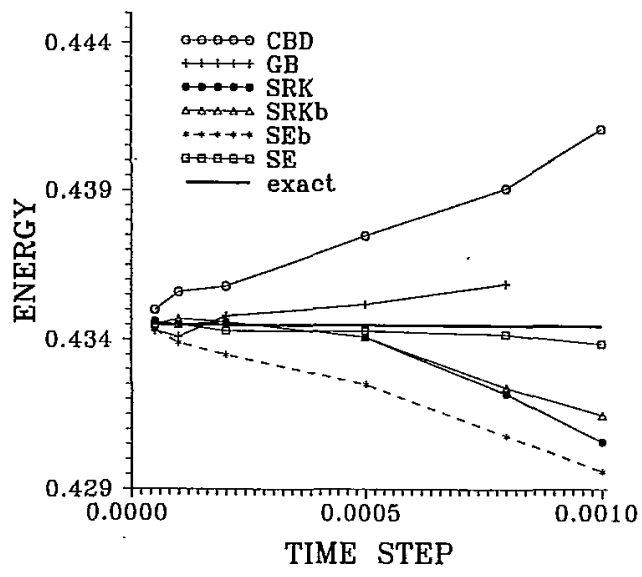

The results produced by the SEb method are only slightly better than that obtained by the CBD one. The GB approach gives fairly good estimation of the energy (the method becomes unstable at $\Delta t>0.0008)$.

\section{CONCLUSIONS}

In the work we have considered algorithms for solving the stochastic differential equation of the position Langevin equations. In particular properties of the Yukawa fluid were analyzed from the point of view of algorithmic efficiency at different time steps.

As expected, the original 1975 first-order Brownian Dynamics algorithm (CBD) due to Ermak is fairly stable but yields the worst estimate of the calculated quantities for any $\Delta t$. With increasing $\Delta t$ it generates a significant overestimation of both static and dynamic quantities. The van Gunsteren and Berendesen, GB, algorithm gives the largest deviation for the mean square displacement in a time step (MSD1) and, as for the CBD method, is not able to reproduce correctly the short-time region of the mean square displacement. Thus, both these algorithms should be used with caution when accurate results for dynamical quantities at short and intermediate times are required. The GB algorithm, however, yields a much better estimate of static quantifies than the CBD algorithm. Such behavior follows probably from the fact that although the GB algorithm is of higher order than the CBD it is not a true second order algorithm as the deterministic part is of order $\Delta t^{2}$ and the stochastic part involves only a term at the $\Delta t^{1 / 2}$ level.

Terms of different levels are involved also in the SEb algorithm (the deterministic part here is of order $\Delta t$ but the stochastic one of $\Delta t^{3 / 2}$ ). Inversly to the GB scheme it gives rather unsatisfactory energy estimation but good short-time msd estimates. The slight bending of the msd at intermediate times makes the $\mathrm{SEb}$ approach rather not competitive to the second order schemes.

The second order position-update schemes considered, the stochastic Runge-Kutta algorithm (SRK), SRKb, and the stochastic equation based on Eq. (8) in the text, give the correct form for the MSD1 and the best estimation for the mean square displacements. The SE approach gives also the best estimation for the energy. The SRKb scheme offers more accurate estimation of calcula- 
ted quantities than the SRK approach. But, the improvement is very marginal and in practice it does not compensate the additional programming efforts required by the extra force terms in the $\mathrm{SRKb}$ update scheme. The form of the error of the MSD1 and its systematic influence on the calculated quantities implies that efficiency of all studied algorithms decreases as the interaction potential becomes less soft.

The differences between the SE and SRK algorithms come from the fact that SRK is an approximation of Eq. (8) in which all of the random terms are represented by a single random number term. Our results suggest that a more rigorous implementation of this expansion in general gives improvements in the accuracy of the calculated quantities. A disadvantage of the SE method is that higher order derivatives of the interaction potential are involved in the calculation and evaluation of correlated random numbers is necessary. Also an extension of the SE approach to deal with position-dependent diffusion coefficients seems to be rather difficult as the stochastic part of the expansion becomes prohibitively complicated. Our calculations indicate that the large number of rather complicated force related terms in the SE scheme increases considerably computational requirements per step. We estimate its computational efficiency between the CBD and SRK methods. The SE approach should have advantage over other approaches in the case of a few variable problems and/or simple form of the interparticle interactions (e.g., the harmonic interactions).

\section{Acknowledgment}

The work has been supported by the Polish Committee for Scientific Research (KBN) grant No. 8T11F01214. Some of the calculations were performed at the Poznań Computer and Networking Center.

\section{References}

[1] D. L. Ermak, J. Chem. Phys., 62, 4189 (1975).

[2] D. L. Ermak, J. A. McCammon, J. Chem. Phys., 69, 1352 (1979).

[3] E. Helfand, J. Chem. Phys., 69, 1010 (1978).

[4] A. Iniesta, J. Garcia de la Torre, J. Chem. Phys., 92, 2015 (1990).

[5] R. L. Honeycutt, Phys. Rev. A 45, 600 (1992).

[6] W. F. van Gunsteren, H. J. C. Berendsen, Molec. Phys., 45, 637 (1982).

[7] X. Y. Chang, K. F. Freed, Chem. Engin. Science, 49, 2821 (1994).

[8] R. J. A. Tough et al., Molec. Phys., 59, 595 (1986).

[9] A. Greiner, W. Strittmatter, J. Honerkamp, J. Stat. Phys., 51, 95 (1988).

[10] W. Hess, R. Klein, Adv. Phys., 32, 173 (1983).

[11] H. LSwen, Phys. Rev. Lett., 72, 424 (1994); J. Chem. Phys., 100, 6738 (1994), 